\title{
I-DTI: a second opinion platform between healthcare professionals related to organ donation and transplantation
}

\author{
Chloe Balleste ${ }^{1}$, Estephan Arredondo², Marian Irazabal ${ }^{2}$, Carlos López $^{2}$, Jordi Colomer ${ }^{2}$, Maria Paula Gómez², \\ Martí Manyalich²

\footnotetext{
1Department of International Cooperation \& Development, Donation and Transplantation Institute (DTI Foundation), Barcelona, Spain

${ }^{2}$ Department of Tissue and Cells, Donation and Transplantation Institute (DTI Foundation), Barcelona, Spain
}

Background: Due to COVID-19 pandemic, organ donation and transplantation (ODT) rates have significantly decreased worldwide. Social and displacement restrictions, along with safety measures proved even more how necessary telematic communications are. Designed as a second opinion platform, I-DTI allows to share knowledge between healthcare professionals and internationally recognized experts related to ODT.

Methods: I-DTI is developed based on Medxat ${ }^{\circledR}$ app, accessible via www.i-dti.com and downloadable from digital stores for mobile devices. Database server is encrypted with RAS using cloud technologies. Information is sent through HTTPS (SSL/TLS) codified channels complying data protection laws. I-DTI main function is 24/7 consultancy, covering the following topics: organ donation, transplantation and follow-up, tissue donation and COVID-19. Also, with social network features like instant messaging, profile customization and finder. In order to develop, assess and validate I-DTI platform, from April to December 2020 a pilot phase was performed. I-DTI services, contents, accessibility, and other quality indexes were evaluated in participant hospitals from countries such as India, Philippines, Sri Lanka, Trinidad and Tobago. Workshops were arranged to display I-DTI and teach the basics to more than 60 professionals.

Results: During this period, an average of five consultancies per week were received and answered in less than 24 hours considering urgency and study deepness. COVID-19 related consultancies represented over $50 \%$ of total. All data compilated was anonymized and stored in library cases for academic purposes. In order to study quality indexes, satisfaction surveys were delivered to users, receiving an average score of 9.3/10.

Conclusions: I-DTI proved great value for knowledge sharing, international cooperation, and data compilation especially in developing countries. The specialization of I-DTI opens a way to develop new contents and to partner with technological entities to introduce technological solutions like artificial intelligence and chat bot. So far, more than 650 users from more than 20 countries (65\% in developing) joined I-DTI.

Corresponding author: Chloe Balleste

E-mail: chloe.balleste@dtifoundation.com

\footnotetext{
(c) The Korean Society for Transplantation

This is an Open Access article distributed under the terms of the Creative Commons Attribution Non-Commercial License (http://creativecommons.org/licenses/by-nc/4.0/) which permits unrestricted non-commercial use, distribution, and reproduction in any medium, provided the original work is properly cited.
} 